\title{
A Low-Power High-Speed Smart Sensor Design for Space Exploration Missions
}

\author{
Wai-Chi Fang \\ Jet Propulsion Laboratory, California Institute of Technology \\ 4800 Oak Grove Drive, Pasadena, CA 91109-8099, USA
}

\section{ABSTRACT}

A low-power high-speed smart sensor system based on a large format active pixel sensor (APS) integrated with a programmable neural processor for space exploration missions is presented. The concept of building an advanced smart sensing system is demonstrated by a system-level microchip design that is composed with an APS sensor, a programmable neural processor, and an embedded microprocessor in a SOI CMOS technology. This ultra-fast smart sensor system-on-achip design mimics what is inherent in biological vision systems. Moreover, it is programmable and capable of performing ultra-fast machine vision processing in all levels such as image acquisition, image fusion, image analysis, scene interpretation, and control functions. The system provides about one tera-operation-per-second computing power which is a two order-ofmagnitude increase over that of state-of-the-art microcomputers. Its high performance is due to massively parallel computing structures, high data throughput rates, fast learning capabilities, and advanced VLSI system-on-a-chip implementation.

\section{INTRODUCTION}

In this paper, an ultra-fast low-power smart vision system-on-a-chip design is proposed to provide effective solutions for real time machine vision applications by taking advantages of advances in integrated sensing/processing designs, electronic neural networks, advanced microprocessors, and sub-micron VLSI systems-on-a-chip (SOAC) technology. The proposed smart vision system mimics what is inherent in biological vision systems. Moreover, this vision system is programmable and capable of performing ultra-fast machine vision processing in all levels such as image acquisition, image fusion, image analysis, scene interpretation, and control functions. The system provides about one tera-operation-per-second computing power which is a two order-of-magnitude increase over that of state-of-the-art microcomputers. Its high performance is due to massively parallel computing structures, high data throughput rates, fast learning capabilities, and advanced VLSI system-on-a-chip implementation. This highly integrated smart vision system can be used for various space exploration missions. For example, it can be used as a smart navigation sensor that is required for autonomous rendezvous and docking.

\section{SMART VISION PROCESSING ALGORITHM AND ARCHITECTURE}

The human visual system provides a demonstration of a versatile and powerful vision system [1]. In general, much of the current machine vision research concerns not only understanding the process of vision but also designing effective vision systems for various real-world applications [2]. 
The proposed versatile vision system is based on a simplified model of the human visual system as shown in Figure 1 [3]. The ultimate design goal is to build an eye-brain machine which can automatically recognize, localize, and classify point, area and volume objects and phenomena in real-time. In general, the eye-brain machine (EBM) includes two major subsystems: the EBM Eye and the EBM Brain. The EBM Eye is a compact optoelectronic subsystem which integrates a wide range of different sensors with geometric, radiometric, and spectral parameters meeting the actual science and mission requirements. The EBM Brain is a high performance control and data handling subsystem, which provides computing resources to perform various on-board vision tasks.

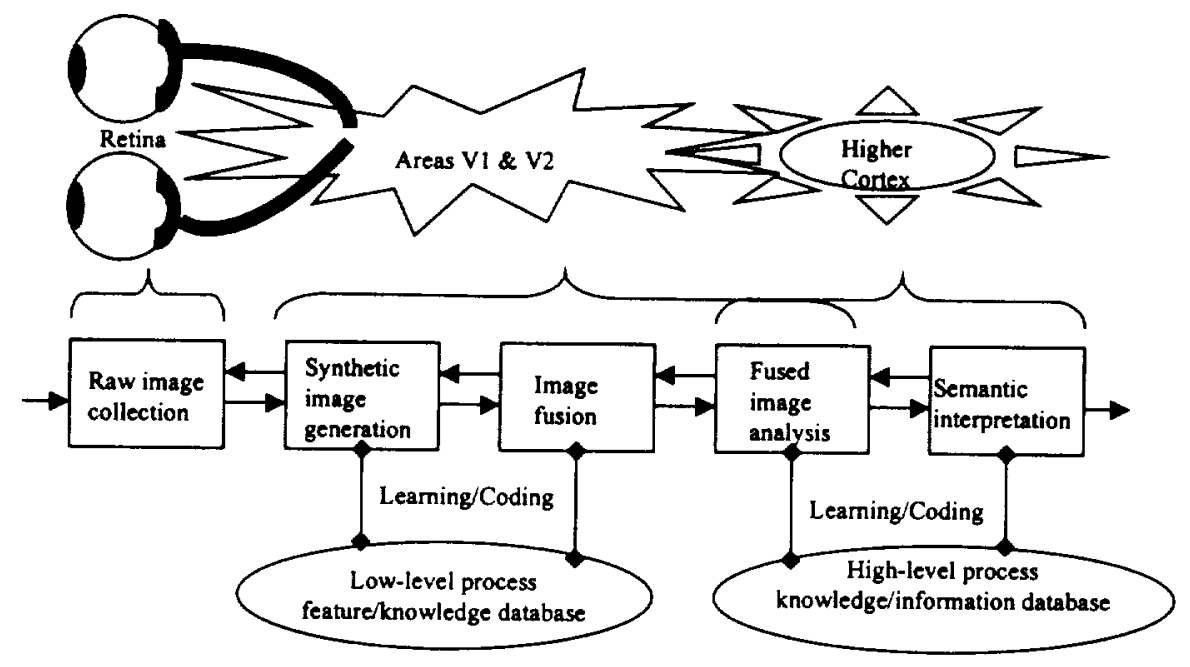

Figure 1. Smart vision processing model based a simplified human visual process flow.

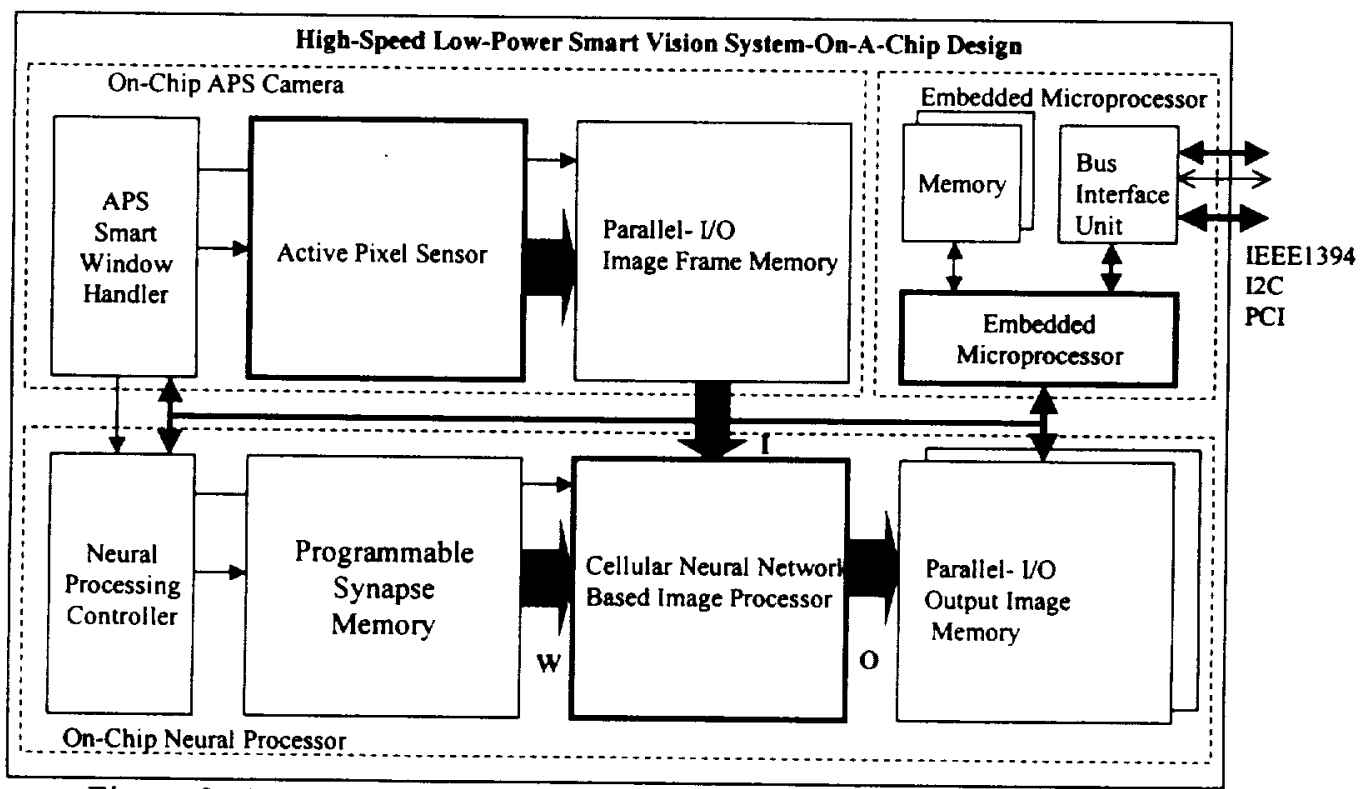

Figure 2. A system diagram of the smart vision system-on-a-chip design.

Figure 2 shows a system diagram of the proposed smart vision system-on-a-chip design. An onchip row/column-parallel image flow architecture is used to connect all on-chip systems and eliminate 
data bandwidth bottlenecks due to conventional bus architectures. The on-chip APS imager is used as the optical sensing array in the system. Windowed image data is fed to the on-chip neural neural processor under the control of a smart window handler. The on-chip neural computer is programmed to perform various early vision tasks in high speed. This tera-operation-per-second neural processor serves as a supercomputing engine for various vision processing tasks due to its massively parallel computing structures and its programmability. The on-chip microcomputer is used to perform command and control of the system operation and scene interpretation. The vision system can work with a remote host system through the bus interface unit.

A system-on-a-chip implementation of this smart vision system is shown to be feasible by integrating the whole system into a $2-\mathrm{cm} \times 2-\mathrm{cm}$ chip design in a $0.18-\mu \mathrm{m}$ SOI CMOS technology [4] (see Table 1). The SOAC-based vision system design has advantages over the COTS-based design such as higher on-chip system integration, $10 \mathrm{x}$ improvement in system miniaturization, 10x lower power dissipation, and $\mathrm{xl} 0$ better performance.

Table 1: Area and power estimates for the smart vision system design in a $0.18-\mu \mathrm{m}$ CMOS technology.

\begin{tabular}{|l|l|r|r|}
\hline Smart Vision Processor & Technology: 0.18- $\mu \mathrm{m}$ SOI CMOS & Vdd =0.9 Volt \\
\hline On-Chip Systems & Building Blocks & Size (mm sq.) & Power (mW) \\
\hline On-Chip Camera & APS Sensor (1024x1024) & 16 & 30 \\
\hline & Image Frame Memory (1MBytes) & 16 & 50 \\
\hline & Smart Window Handler & 9 & 40 \\
\hline On-Chip Neural Computer & $\begin{array}{l}\text { Neural Processor (1024x1024 or } \\
\text { 64x64x64) }\end{array}$ & 64 & 200 \\
\hline & Synapse Memory (1MBytes) & 16 & 50 \\
\hline & Activity Memory (1MBytes) & 9 & 50 \\
\hline & Neural Processing Controller & 45 & $200 @ 300 \mathrm{MHz}$ \\
\hline On-Chip Microcomputer & Microprocessor & 144 & 100 \\
\hline & Memory (32 Mbytes) & 16 & $\mathbf{3 3 5}$ \\
\hline & Bus Interface and Control & $\mathbf{7 9 0}$ \\
\hline & Total: & & 40 \\
\hline
\end{tabular}

\section{ON-CHIP ACTIVE PIXEL SENSOR DESIGN}

High performance CMOS active pixel sensor technology has been developed by NASA's Jet Propulsion Laboratory for electronic image capture [5]. A 1024×1024 CMOS APS prototype chip using $0.5 \mu \mathrm{m} n$-well process was designed and characterized at JPL. It contains a 1024×1024-photogate array and 1024 parallel 10-bit singles-slope ADC. Testing results show that the large format APS with small feature size is capable of excellent imaging performance. A reusable on-chip APS imager based on this prototype APS chip design has been under development in a $0.18 \mu \mathrm{m}$ SOI CMOS technology [6].

The on-chip APS imager is based on a row/column/window-parallel data flow architecture. It includes an APS active sensor, a smart window handler, and a parallel-I/O image frame memory. The smart image window handler is designed for the row/column/window-parallel interface between the APS imager and the neural processor to achieve an ultra-fast frame time up to 1000 frames per second. 


\section{ON-CHIP PROGRAMMABLE NEURAL PROCESSOR DESIGN}

An on-chip programmable neural processor design is used as a parallel processor to perform various vision functions at very high speed. Incorporating the neural computer into the proposed vision system offers orders-of-magnitude computing performance enhancements for real-time vision tasks.

The programmable neural processor is based on optimization cellular neural network (OCNN) [7]. The OCNN is an improved version of the Cellular Neural Networks (CNN) [8]. The CNN has been proved to be universal as the Turing machine. The OCNN keeps this highly desired programmability and become a versatile vision processor. Many OCNN functions have been verified via system simulation. These functions include morphological operations, feature extraction, motion detection, path tracking, collision avoidance, etc. The OCNN architecture is a multi-dimensional array of mainly identical cells, which are dynamic systems with continuous state variables and locally connected with their local cells within a finite radius. A prototype chip of the OCNN was designed and tested [7]. An on-chip OCNN neural processor based on this prototype OCNN chip has been under development for the proposed smart vision system. The parallel column/row/window readout capability is also integrated with the OCNN to accommodate the applications that require high-speed parallel image I/O.

\section{CONCLUSION}

A high-speed low-power smart vision system-on-a-chip design is proposed to provide effective solutions for space exploration missions. A system-on-a-chip implementation of this smart vision system is shown to be feasible by integrating the whole system into a $2-\mathrm{cm} \times 2-\mathrm{cm}$ chip design in a $0.18-\mu \mathrm{m}$ CMOS technology. An on-chip row/column-parallel data flow architecture is used to connect all on-chip systems and eliminate data bandwidth bottlenecks due to conventional bus architectures. This highly integrated smart vision system-on-a-chip design can be used on various space exploration missions and other industrial or commercial vision applications.

\section{ACKNOWLEDGMENTS}

The research described in this paper was performed by the Jet Propulsion Laboratory, California Institute of Technology, and was sponsored by the National Aeronautics and Space Administration. The author would like to thank Drs. L. Alkalai, G. Yang, M. Pain, S. Udomkesmalee and Professor B. Sheu for valuable technical discussions and supports.

\section{REFERENCES}

[1] D. Marr, Vision, Freeman, San Francisco, 1982.

[2] Carver Mead, “ Analog VLSI and Neural Systems," Addison-Wesley Publishing Company, 1989.

[3] Suraphol Udomkesmalee, Anil Thakoor, Curtis Padgett, Taher Daud, Wai-Chi Fang, "VIGILANTE: An advanced sensing/processing testbed for ATR applications," SPIE Orlando, FL, April 21-24, 1997.

[4] Advanced Silicon Technology, MIT Lincoln Laboratory, http://www.ll.mit.edu/AST/

[5] E.R. Fossum, CMOS Image Sensors: Electronic Camera on a Chip (Plenary paper), Tech. Dig. of IEEE International Electron Devices Meeting, pp. 17-25, Dec. 1995.

[6] Wai-Chi Fang, Guang Yang, Bedabrata Pain, "A Low Power Smart Vision System based on Active Pixel Sensor Integrated with Programmable Neural Network Processor," 1997 International Conference on Computer Design, Austin, Texas, Oct. 11-14, 1997.

[7] B. J. Sheu, S. Bang, W.-C, Fang, "VLSI design of cellular neural networks with annealing and optical input capabilities," Proceedings of the IEEE International Symposium of Circuit and System, April 1995.

[8] L. O. Chua, L. Yang, "Cellular neural networks: Theory", IEEE Trans. on Circuits and Systems, Oct. 1988. 